\title{
Simultaneous interrogation of interferometric and Bragg grating sensors
}

\author{
G. Brady, K. Kalli, D. J. Webb, and D. A. Jackson \\ Applied Optics Group, Physics Laboratory, University of Kent at Canterbury, Canterbury, CT2 7NR, UK \\ L. Reekie and J. L. Archambault \\ Optoelectronics Research Centre, University of Southampton, Southampton, Hants, SO9 5NH, UK
}

Received January 23, 1995

\begin{abstract}
We propose a new method for the simultaneous interrogation of conventional two-beam interferometers and Bragg grating sensors. The technique employs an unbalanced Mach-Zehnder interferometer illuminated by a single low-coherence source, which acts as a wavelength-tunable source for the grating and as a path-matched filter for the Fizeau interferometer, thus providing a high phase resolution output for each sensor. The grating sensor demonstrates a dynamic strain resolution of $\sim 0.05 \mu \varepsilon / \sqrt{\mathrm{Hz}}$ at $20 \mathrm{~Hz}$, while the interferometric phase resolution is better than $1 \mathrm{mrad} / \sqrt{\mathrm{Hz}}$ at $20 \mathrm{~Hz}$, corresponding to an rms mirror displacement of $0.08 \mathrm{~nm}$.
\end{abstract}

Bragg grating sensors ${ }^{1}$ (BGS's) have generated interest in recent years because of their potential use in a wide range of applications, particularly for monitoring the structural integrity of buildings and bridges and for use in the aerospace industry, where determination of the fatigue loading of advanced composite materials is essential. BGS's form an integral part of the optical fiber structure, being written intracore (possibly while the fiber is manufactured), and provide absolute wavelength encoding of information that is independent of overall system light levels but dependent on strain and temperature effects acting on the sensor. The small size and the dielectric properties of the fiber make it particularly suited for the above uses. The BGS has other advantages that are similar to those already recognized for existing interferometric point sensors-immunity to electromagnetic interference, high sensitivity, and the potential for multiplexing. ${ }^{2}$

A fiber network able to incorporate both two-beam interferometric point and grating sensors can prove useful in situations for which temperature and strain effects need to be measured separately or for which one measurand needs to be compensated. For example, the BGS can provide complementary temperature information for a Fizeau cavity pressure sensor that is exposed to pressures at high temperatures.

In this Letter we present preliminary data for a system in which a Fizeau cavity and a BGS are operated simultaneously; the sensor's operation is facilitated by an unbalanced Mach-Zehnder interferometer illuminated with a low-coherence source. The Mach-Zehnder interferometer serves to filter the source spectrum with a sinusoidal modulation function, producing a channeled spectrum. ${ }^{3}$ This modulation is essentially maintained in transmission through the fiber network. If the interferometer path imbalance is linearly ramped, the peak output wavelengths are swept in time. When the modulation is incident on the BGS it acts as a wavelength-tunable source; the sinusoidal modulation function convolved with the grating transmission response produces a periodic intensity modulation in the reflected light-a carrier signal that is phase modulated when a signal is applied directly to the grating. To permit absolute, unambiguous sensing one must limit the maximum expected strain level applied to the BGS to one free spectral range of the channeled spectrum, thus limiting any induced phase change to $\pm \pi \mathrm{rad}$. For example, for an optical path difference of $150 \mu \mathrm{m}$, the interferometer wavelength-to-phase-shift responsivity is $0.392 \mathrm{rad} / \mathrm{nm}$, resulting in a strain-tophase-shift responsivity of $4.5 \times 10^{-4} \mathrm{rad} / \mu \varepsilon$ for a grating operated at $1.55 \mu \mathrm{m} .{ }^{4}$ Thus, for a phase change limited to $\pm \pi \mathrm{rad}$, one is limited to a maximum strain for the grating in the range of $\pm 7000 \mu \varepsilon( \pm 7 \mathrm{~m} \varepsilon)$.

The Mach-Zehnder interferometer may also be coherently tuned to a conventional two-beam interferometer, such as the Fizeau cavity used in our experiments. The path-matching condition necessary for interference effects to be observed requires that the unbalanced interferometers have an approximately equal optical path difference, with one interferometer acting as the sensor and the other as the receiver in a composite sensor system. ${ }^{5}$ Illumination with a source having a coherence length far less than the individual interferometer path imbalances does not result in fringes from either interferometer. However, the receiver section provides optical path length compensation for a portion of the received power, and so interference fringes are observed for the composite system but with a reduction in fringe visibility. Coherence tuning is often advantageous, as it allows for the sensing interferometer to remain entirely passive, with any active components located in the receiving interferometer. We have combined these two well-known techniques to recover high-resolution phase information for both types of sensor.

The experimental arrangement is shown in Fig. 1. The fiber network was illuminated with an optically isolated erbium-doped fiber amplifier as a superfluorescent source, producing approximately $100 \mu \mathrm{W}$ of output power within a $30-\mathrm{nm}$ bandwidth $(1530-1560 \mathrm{~nm})$. The source spectrum had two domi- 


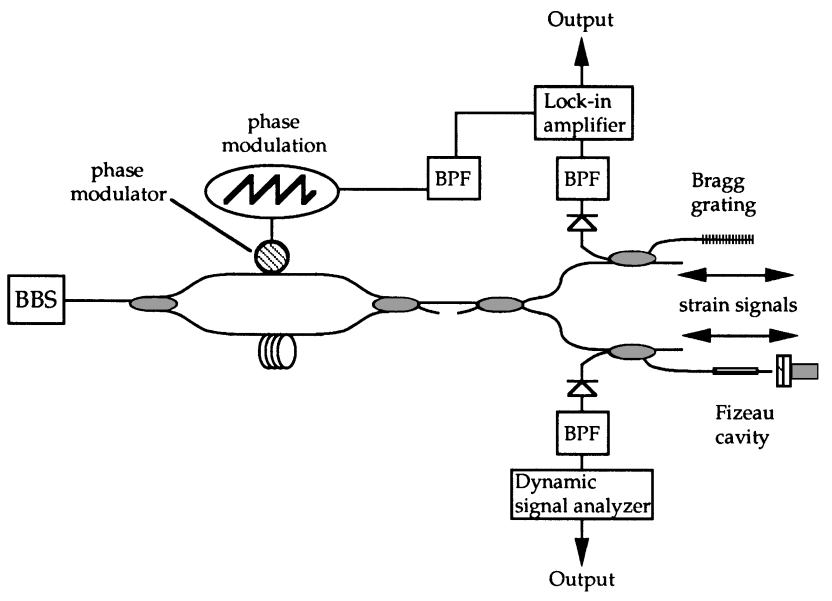

Fig. 1. Interferometric/BGS system using an unbalanced Mach-Zehnder interferometer as a wavelength-tunable source for the grating and as a path-matched filter for the Fizeau interferometer. BBS, broadband source; BPF's, bandpass filters.

nant peaks, located at $1541 \mathrm{~nm}$ (bandwidth $\sim 6 \mathrm{~nm}$ ) and $1535 \mathrm{~nm}$ (bandwidth $\sim 3 \mathrm{~nm}$ ). The fiber source was incident upon an unbalanced fiber Mach-Zehnder interferometer constructed from 50:50 couplers that possessed two arms, each approximately $5 \mathrm{~m}$ in length. The fiber path imbalance corresponded to an optical path difference of $1.5 \mathrm{~mm}$. One fiber arm incorporated a piezoelectric transducer (PZT) cylinder around which was wrapped $\sim 3 \mathrm{~m}$ of fiber; fiber from the other arm was stretched between two translation stages; thus we could modulate the interferometer by applying a signal to the PZT and alter its free spectral range by longitudinally straining the fiber by as much as $1 \mathrm{~cm}$. The output leg of the Mach-Zehnder interferometer was fusion spliced to a $3-\mathrm{dB}$ coupler, the output arms of which were further spliced to another pair of 3-dB couplers. A BGS was placed in the output arm of one coupler and was mounted on a PZT element, permitting a known dynamic strain signal to be applied to the sensor. The grating had a reflection coefficient of $55 \%$ and a $0.2-\mathrm{nm}$ bandwidth at a nominal wavelength of $1534.8 \mathrm{~nm}\left(\right.$ at $25^{\circ} \mathrm{C}$ ); it was specifically chosen to coincide with one of the peak spectral features of the erbium-doped fiber amplifier. For an optical path difference of $1500 \mu \mathrm{m}$, the interferometer wavelength-to-phase-shift responsivity was $3.99 \mathrm{rad} / \mathrm{nm}$, resulting in a strain-to-phase-shift responsivity of $4.5 \times 10^{-3} \mathrm{rad} / \mu \varepsilon$ for this grating and a maximum strain range of $\pm 700 \mu \varepsilon$.

A Fizeau cavity was formed between the fiber end face of one arm of the second coupler and an external mirror mounted on a PZT. The air cavity was path matched to the Mach-Zehnder interferometer, forming a coherence-tuned composite interferometer. The sensor signals were detected with two InGaAs photodiodes, permitting separate recovery of the wavelength-dependent phase shifts from each sensor. The overall gain of each photodetector plus amplifier was $17 \times 10^{7} \mathrm{~V} / \mathrm{W}$, with a noise floor of $26 \mu \mathrm{V} / \sqrt{\mathrm{Hz}}$ at $1 \mathrm{kHz}$. The average optical power at each detector corresponded to $\sim 80 \mathrm{nW}$.
A serrodyne modulation signal at a frequency of $1 \mathrm{kHz}$ was applied to the Mach-Zehnder interferometer, producing a peak-to-peak phase excursion of $2 \pi \mathrm{rad}$. Thus the interferometer simultaneously acted as a wavelength-tunable source for the BGS and a coherence-tuned receiving interferometer for the Fizeau cavity. The fringe visibility of the output from the grating sensor was $\sim 0.2$, while that from the Fizeau cavity was $\sim 0.27$. A $20-\mathrm{Hz}$ sinusoidal test signal was applied to the BGS. We bandpass filtered the photodetected output at the carrier modulation frequency and directly compared its phase with the constant phase of the carrier signal using a lock-in amplifier, the output of which was recovered with a storage oscilloscope and dynamic signal analyzer. Similarly, the Fizeau cavity mirror was modulated at $20 \mathrm{~Hz}$ with a sinusoidal signal; the interferometric information was recovered directly with a dynamic signal analyzer.

Figure 2 shows the spectrum recovered when the Fizeau cavity was subject to an rms displacement of $7.6 \mathrm{~nm}$ at $20 \mathrm{~Hz}$; the signal-to-noise ratio is $40 \mathrm{~dB}$ in

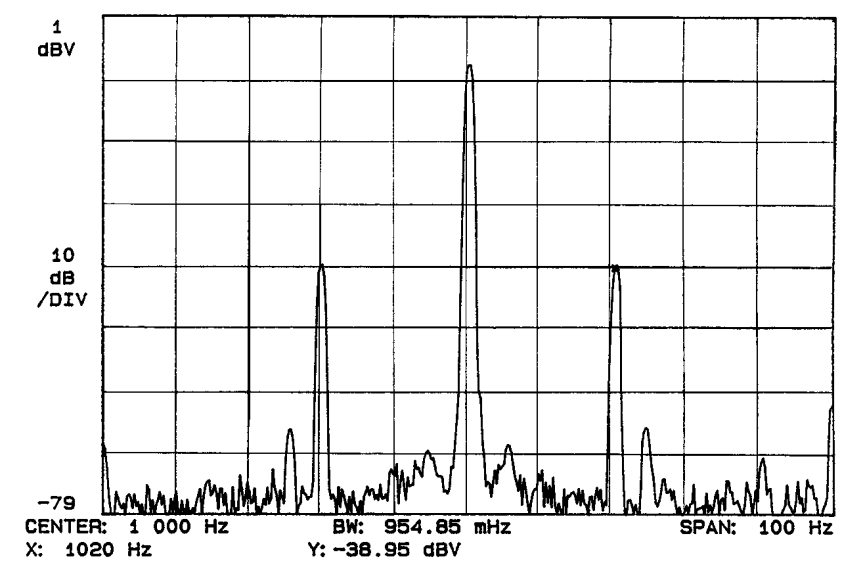

Fig. 2. Power spectrum from the coherence-tuned interferometers (centered at $1 \mathrm{kHz}$ ) with the Fizeau cavity subject to an $\mathrm{rms}$ displacement of $7.6 \mathrm{~nm}$ at $20 \mathrm{~Hz}$. Vertical scale, $10 \mathrm{~dB} /$ division; horizontal scale, $10 \mathrm{~Hz} /$ division.

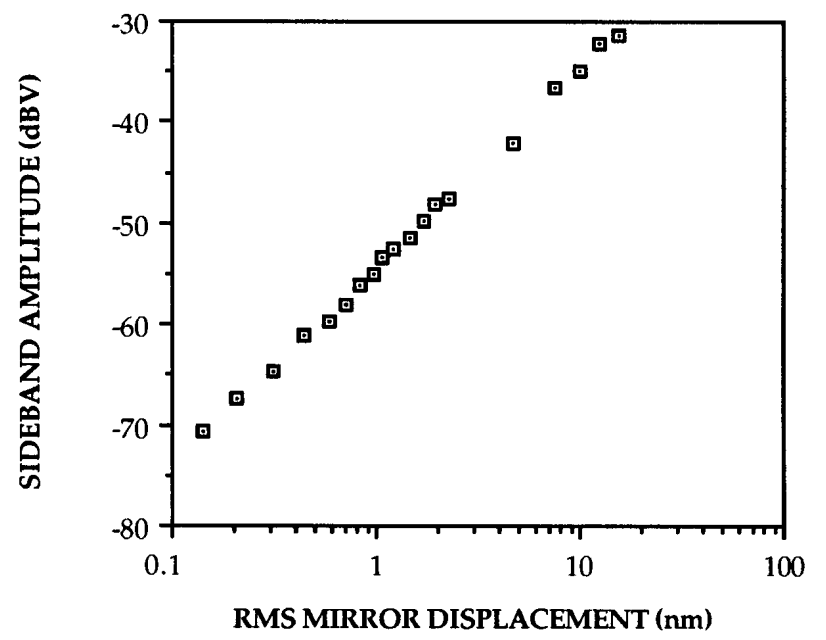

Fig. 3. Linearity of the coherence-tuned interferometers to rms displacements of the Fizeau cavity mirrors. 


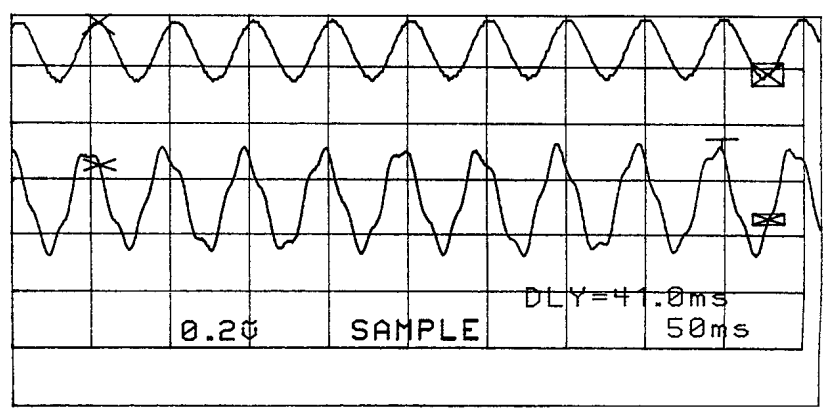

Fig. 4. Upper trace: Test signal producing an rms mirror displacement of $12.5 \mathrm{~nm}$ at $20 \mathrm{~Hz}$ applied to the Fizeau cavity. Lower trace: Lock-in amplifier output response of the Fizeau cavity. The output is modulated by acoustic pickup of the Mach-Zehnder interferometer. Vertical scale, arbitrary units; horizontal scale, $50 \mathrm{~ms} /$ division.

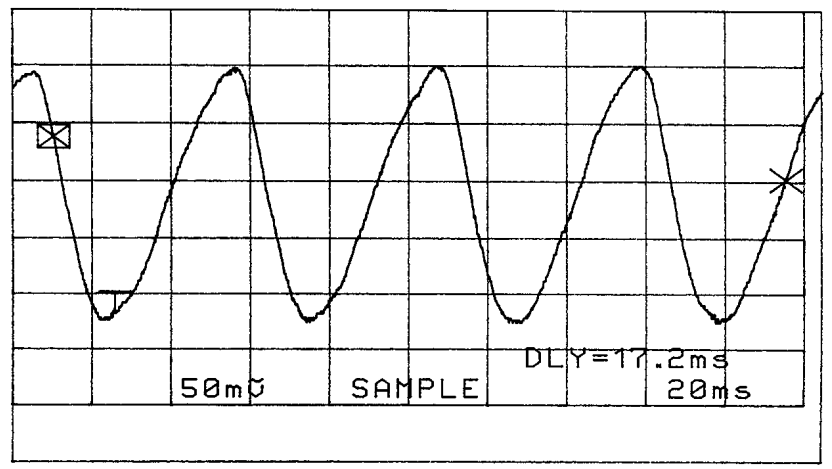

Fig. 5. Lock-in amplifier output response of the Bragg grating sensor to a peak-to-peak strain of $\sim 18 \mu \varepsilon$ at $20 \mathrm{~Hz}$. Vertical scale, arbitrary units; horizontal scale, $20 \mathrm{~ms} /$ division.

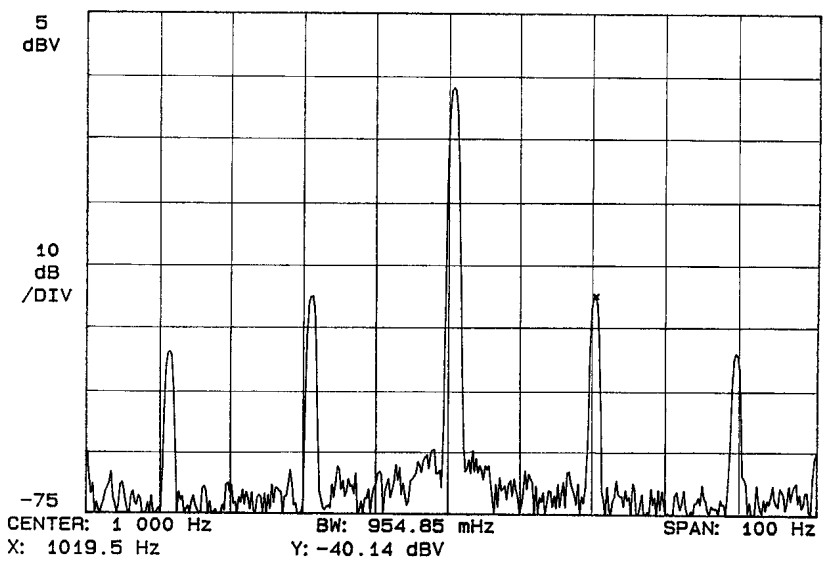

Fig. 6. Power spectrum of the Bragg grating sensor subject to a peak-to-peak strain of $\sim 18 \mu \varepsilon$ at $20 \mathrm{~Hz}$. Significant $40-\mathrm{Hz}$ components are present as a result of hysteresis present in the PZT element attached to the grating. Vertical scale, $10 \mathrm{~dB} /$ division; horizontal scale, $10 \mathrm{~Hz}$ /division.

a $0.955-\mathrm{Hz}$ bandwidth, equivalent to a minimum detectable displacement of $0.078 \mathrm{~nm}$ when normalized to a $1-\mathrm{Hz}$ bandwidth. Figure 3 represents displacement measurements over the range $0.14-15.47 \mathrm{~nm} \mathrm{rms}$ and shows reasonable linearity.
The lower trace of Figure 4 shows the output from the lock-in amplifier for an rms mirror displacement of $12.5 \mathrm{~nm}$; the upper trace represents the signal applied to the mirror. The signal is modulated by a strong $75-\mathrm{Hz}$ component-a result of acoustic pickup by the Mach-Zehnder interferometer.

A peak-to-peak strain of $17.95 \mu \varepsilon$ was applied to the Bragg grating at a frequency of $20 \mathrm{~Hz}$; the appropriate lock-in amplifier output is displayed in Fig. 5. The signal is not perfectly sinusoidal, showing the hysteresis present in the PZT to which the grating was attached. This is further indicated in the spectrum analyzer trace of Fig. 6 , for which significant $40-\mathrm{Hz}$ components are present in addition to the $20-\mathrm{Hz}$ frequency components. To investigate the sensitivity of the system to small strain perturbations we applied a $\sim 0.6-\mu \varepsilon \mathrm{rms}$ signal-again at $20 \mathrm{~Hz}$. From the power spectrum of the phase difference output of the lock-in amplifier the signal-to-noise ratio at $20 \mathrm{~Hz}$ normalized to a $1-\mathrm{Hz}$ bandwidth corresponded to a minimum detectable strain perturbation of $\sim 50 \mathrm{n} \varepsilon / \sqrt{\mathrm{Hz}}$.

In conclusion, we have successfully demonstrated the simultaneous operation of a two-beam interferometric point sensor and a BGS, utilizing a single Mach-Zehnder interferometer, illuminated by a lowcoherence source, as a phase discriminator for both sensors. We see this as an important development. Both BGS and low-coherence interferometric sensors are currently of considerable interest to a number of industries. The fact that both sensor types can be incorporated within the same system may be very attractive for some applications. Note that, while for demonstration purposes we have chosen to interrogate one sensor of each type, in principle many sensors could be addressed by the same reference interferometer. The sensors are only as stable as the MachZehnder interferometer; a dramatic improvement in the measured low-frequency response would be realized if that interferometer were implemented as an unbalanced integrated-optic device. The small path imbalance would increase the dynamic range of the grating and reduce the effects of phase noise on the optical phase recovered by the two-beam interferometer. The grating sensor demonstrated a dynamic strain resolution of $\sim 0.05 \mu \varepsilon / \sqrt{\mathrm{Hz}}$ at $20 \mathrm{~Hz}$, while the interferometric phase resolution was better than $1 \mathrm{mrad} / \sqrt{\mathrm{Hz}}$ at $20 \mathrm{~Hz}$, corresponding to an $\mathrm{rms}$ mirror displacement of $0.08 \mathrm{~nm}$.

\section{References}

1. G. Meltz, W. W. Morey, and W. H. Glenn, Opt. Lett. 14, 823 (1989).

2. A. D. Kersey, Proc. Soc. Photo-Opt. Instrum. Eng. 2071, 30 (1993).

3. D. A. Norton, Proc. Soc. Photo-Opt. Instrum. Eng. 1795, 371 (1992).

4. A. D. Kersey, T. A. Berkoff, and W. W. Morey, Opt. Lett. 18, 72 (1993).

5. F. Farahi, T. P. Newson, J. D. C. Jones, and D. A. Jackson, Opt. Commun. 65, 319 (1988). 